\title{
Beneficios de la investigación médica aplicados a los niveles de prevención
}

\author{
Benefits of medical research applied to levels of prevention \\ Mayra Contreras-León ${ }^{a}$, Grecia Jarillo-Domínguez ${ }^{b}$, José M. Alonso-Molina $^{c}$
}

\begin{abstract}
:
Research is the basis of all existing science; it is essential to keep concepts and knowledge updated so that they are useful in our daily lives, an example of this is medicine that is modernized in several ways such as with new findings, updating treatments and with the constant studies that are done to try to solve many doubts that still have no answer or to delay any of the processes of the disease as it happens in the application of the levels of preventive and that there has been no better treatment for some diseases than prevention and it is very useful to generate new forms of care to keep the population healthy since this has a direct impact on the quality and life expectancy of people.
\end{abstract}

Keywords:

Prevention, benefits, medicine, research

\section{Resumen:}

La investigación es la base de toda ciencia existente; resulta fundamental mantener actualizados los conceptos y conocimientos para que resulten útiles en nuestra vida cotidiana, un ejemplo de ello es la medicina que se moderniza de varias maneras como con nuevos hallazgos, actualizando los tratamientos y con los constantes estudios que se hacen para intentar resolver muchas dudas que aún no tienen respuesta o para retrasar alguno de los procesos de la enfermedad como sucede en la aplicación de los niveles de preventivos ya que no ha existido mejor tratamiento para algunas enfermedades que la prevención y resulta muy útil la generación de nuevas formas de atención para mantener saludable a la población ya que esto tiene un impacto directo en la calidad y esperanza de vida de las personas.

\section{Palabras Clave:}

Prevención, beneficios, medicina, investigación

\section{Introducción}

Los niveles de prevención se definen como estrategias para retrasar la aparición de una enfermedad o sus efectos por lo que actúan en distintas etapas de la historia natural de la enfermedad, entre los cuales podemos reconocer 3 niveles:

1. El nivel primario está dirigido a personas con ausencia de patología, es decir saludables

2. El nivel secundario se refiere a las acciones que se deben realizar cuando comienzan a hacerse evidentes las manifestaciones clínicas y está encaminado a la detección temprana para reducir complicaciones.

3. El tercer nivel se enfoca en la rehabilitación de la persona, buscar evitar secuelas y poder aportar una buena calidad de vida.

La investigación médica tiene por objetivo generar nuevos conocimientos a esta ciencia y puede beneficiar mucho a los niveles de prevención ya que actualmente se intenta entender las enfermedades y crearles tratamientos eficaces (creación de fármacos) por lo que se puede decir que es parte de una medicina preventiva.

\section{Autor de correspondencia, Universidad Autónoma del Estado de Hidalgo, https://orcid.org/0000-0002-6336-1597, Email: co419484@uaeh.edu.mx \\ b Grecia Jarillo Domínguez, Universidad Autónoma del Estado de Hidalgo, https://orcid.org/0000-0002-6157-1259, Email: ja315448@uaeh.edu.mx




\section{Beneficios de la investigación médica aplicados a los niveles de prevención}

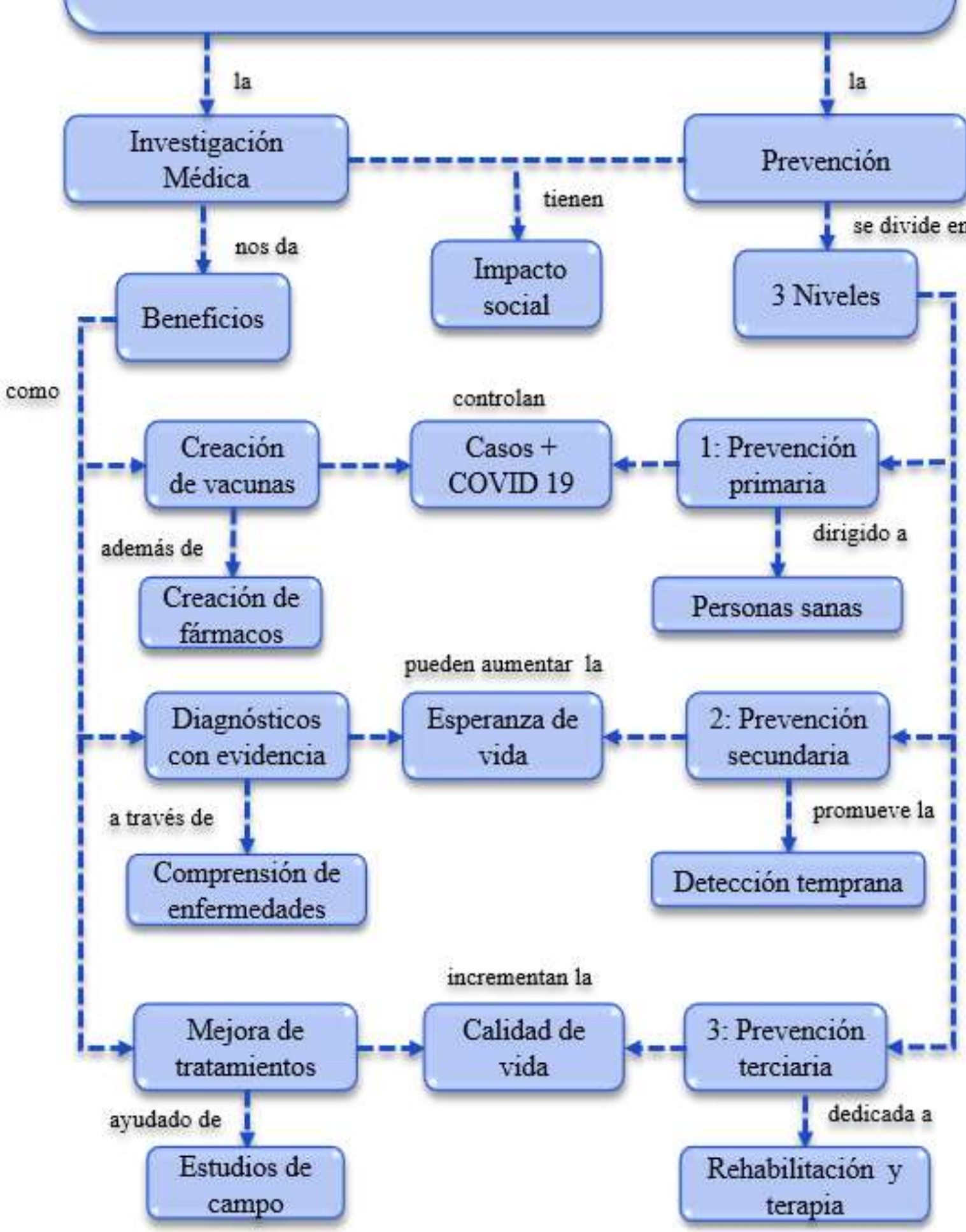




\section{Referencias}

[1] Amando Martín Zurro, A. a. (15 de junio de 2021). Compendio de Atención Primaria (Quinta Edición ed.). Elsevier

[2] America, P. o. (2020). Prevention. Obtenido de https://www.cdc.gov/pictureofamerica/pdfs/picture_of_america_preve ntion.pdf

[3] González, M. Á. (7 de febrero del 2018). Conceptos de Salud Pública y estrategias preventivas

[4] Health, I. F. (abril de 2016). Primary, secondary and tertiary prevention. Obtenido de: https://www.iwh.on.ca/what-researchers-meanby/primary-secondary-and-tertiary-prevention

[5] Instituto Nacional de Ciencias Médicas y Nutrición Salvador Zubirán, I. (13 de junio 2019). ¿Qué es la investigación clínica y porque participar en estudios? Obtenido de: https://www.incmnsz.mx/opencms/contenido/investigacion/subInvesti gacion/investigacion_clinica.html

[6] Manucha, W. (octubre 26 de 2019). La importancia de la investigación científica en medicina. doi:ISSN 0121-0319

[7] OPS. (julio de 2020). OPS (Organización Panamericana de la Salud) Obtenido de Promoción de la Salud

[8] OPS (Organización Panamericana de la Salud). (2020). Atención primaria de salud. Obtenido de: https://www.who.int/es/newsroom/fact-sheets/detail/primary-health-care

[9] (Organization), P. (. (2015). Population an individual Approaches to the prevention and Management of diabetes and obesity. Obtenido de https://www.paho.org/hq/dmdocuments/2012/DMPLAN-

ENGLISH.pdf

[10] Organization, W. H. (2018). Guide to national implementation

[11] Prevention and Community Health. (29 de septiembre de 2016). Obtenido

http://samples.jblearning.com/9781284090765/Chapter2.pdf

[12] Principled Promotion of Health: Implementing Five guiding health promotion principles for research - based prevention and management of diabetes. (2017). En J. A.-H. Dan Grabowski.

[13] Rey, R. (2016). Investigación Traslacional en Medicina. Buenos Aires

[14]Salud, C. E. (2019). Options to foster health promoting. En Expert Panel on effective ways of investing in Health (EXPH). Unión Europea.

[15] Zvonko Sosic, MD, MSc, PhD, Associate Professor, \& Doncho Donev, MD, PhD, Professor. (2020). Health promotion and disease prevention. Obtenido de: https://core.ac.uk/download/pdf/333548429.pdf 